\title{
Effect of SMES unit in load following contract in a restructured power system
}

\author{
Sandeep Bhongade ${ }^{* 1}$, Barjeev Tyagi ${ }^{2}$, and H.O. Gupta ${ }^{3}$ \\ ${ }^{1 *}$ Department of Electrical Engineering, Indian Institute of Technology Roorkee, INDIA \\ *Corresponding Author: e-mail: bhongadesandeep@gmail.com, Tel +91-1332285555, Fax. +91-1332-286691
}

\begin{abstract}
The purpose of this paper is to analyze the effect of SMES unit on the Dynamic Neural Network (DNN) based multi area AGC scheme. The advantage of the DNN controller is that it does not require extensive and rigorous model for optimal tuning. It requires a set of training data. The training data can be generated from the system model. The developed DNN controller has been tested on a practical Indian power system network representing 75- bus system. A deregulated electricity market scenario has been assumed in the 75- bus system, which has been divided into four control areas. Mixed transaction (Poolco and bilateral) has been considered in the frequency regulation.
\end{abstract}

Keywords: Deregulated market, Dynamic Neural Network, Automatic Generation Control, Area control error.

\section{Introduction}

Automatic generation control (AGC) is a very important problem in power system operation and control. AGC, in Power Systems is the mechanism by which a balance between electric power generation and power consumption is maintained. Modern power system consists of number of control areas interconnected together and power is exchanged between control areas over tie-lines by which they are connected. In order to achieve interconnected operation of a power system, an electric energy system must be maintained at a desired operating level characterized by nominal frequency, voltage profile and load flow configuration. This is achieved by close control of real and reactive powers generated through the controllable source of the system. AGC plays a significant role in the power system by maintaining scheduled system frequency and tie line flow during normal operating condition and also during small perturbations. Many investigations in the area of isolated and interconnected power systems have been reported in the past. The concept of conventional AGC is discussed in Elgerd et al. (1970) and in Jaleeli et al. (1992). Around the world, the electric power industry has been undergoing reforms from the traditional regulated, vertically integrated utility into a competitive, deregulated market. Market deregulation has caused significant changes not only in the generation sector, but also in the power transmission and distribution sectors and has introduced new challenges for market participants. A detailed discussion on Load Frequency Control issues in power system operation after deregulation is reported in Bose et al. (1996).

The application of Superconducting Magnetic Energy Storage (SMES) to electric power systems can be grouped into two categories (Tripathy et al., 1992): (1) large scale energy storage like conventional pumped hydro plant storage meant for diurnal load leveling application and (2) low capacity storage to improve the dynamic performance of the power system.

In the first case, large sized (hundreds of meters in diameter) high capacity superconducting magnets capable of storing $10^{2} \mathrm{MJ}$ are necessary (Peterson et al., 1975). For the second application, very small sized SMES units with storage capacity of the order of $18 \mathrm{MJ}$ or even less would be sufficient. Several design methods of AGC, which is equipped with SMES, have been successfully proposed (Joseph et al. (2007) and Shayeghi et al. (2008).

In this work, an investigation has been carried out the effect of a small sized SMES unit on DNN based multi area AGC scheme. The advantage of the DNN controller is that it does not require extensive and rigorous model for optimal tuning. It requires a set of training data. The training data can be generated from the system model. The developed DNN controller has been tested on a practical Indian power system network representing 75- bus system. A deregulated electricity market scenario has been assumed in 
the 75- bus system, which has been divided into four control areas. Poolco based and bilateral (mixed) transactions have been simulated. The performance studies have been carried out by using the MATLAB SIMULINK for transactions within and across the control area boundaries.

\section{Problem Formulation}

Introduction of competition in electricity market may cause emergence of several new entities, such as Generating companies (Gencos), Transmission companies (Transcos), Distribution companies (Discos) and system operator (SO). The system operator is an entity entrusted with the responsibility of ensuring the reliability and security of the power system. It is an independent entity and does not participate in the electricity trading. In order to maintain the system security and reliability, the SO procures various services, such as supply of emergency reserves, frequency regulation and reactive power from the other entities in the system. These services are known as the 'ancillary services'. In a competitive electricity environment, Poolco as well as bilateral transactions may take place simultaneously.

\section{A. Poolco based transaction}

In Poolco based transaction (Kumar et al, 1997), the Discos and Gencos of the same area participate in the frequency regulation through system operator. System operator (SO) accepts bids (volume and price) from power producers (Gencos) who are willing to quickly (with in about 10-15 minutes) increase or decrease their level of production. Consumers (Discos) also can submit bids to SO for increasing or decreasing their level of consumption. In each hour of operation, the SO activates the most favorable bid.

If the frequency is lower than nominal value, up regulation bids are activated by the System Operator in steps and the highest activated bid becomes the regulation price, uniformly paid to all the providers of upward regulation service. If the frequency is higher than nominal, down regulation is activated by the System Operator in steps and the lowest activated bid price becomes the uniform price, to be paid by all the down regulation service providers. Thus, the hourly regulating price is fixed as the price for the most expensive measure (regulating up) or least expensive measure (regulating down) utilized during the hour. At the end of scheduled interval, the net energy balance of each entity is calculated and financial settlements are carried out.

\section{B. Bilateral transaction}

In bilateral transaction (Kumar et al., 1997, Donde et al, 2001 and Bhattacharya et al, 2000), Gencos and Discos negotiate bilateral contracts among each other and submit their contractual agreements to SO. The players are responsible for having a communication path to exchange contract data as well as measurements to do load following in real-time. In such an arrangement, a Disco sends a pulse to Genco to follow the predicted load as long as it does not exceed the contracted value. The responsibility of the Disco is to monitor its load continuously and ensure the loads following requirements are met according to the contractual agreement.

In this work, bilateral transactions within the area and across the area have been considered. Disco of one area can contract to the Genco of same area or other area to supply a certain amount of power in a specified time interval. These bilateral contracts can be represented in the matrix form in which the number of rows equal to the number of Gencos and column equal to the number of Discos in the system. The elements of this Contract Participation Matrix (CM) represent the percentage load demand of one Disco to different Gencos. Let us consider a Contract Matrix as given below:

$$
C M=\left[\begin{array}{ccccc}
0 & 10 & . & . & . \\
20 & 10 & . & . & . \\
10 & . & . & . & . \\
. & . & . & . & . \\
. & . & . & . & . \\
0 & 0 & . & . & .
\end{array}\right]
$$

For example, the first column of CM represents the Disco D1 bilateral contract with different Gencos. Element $\mathrm{CM}_{21}$ is 20 which mean $20 \%$ of total demand of Disco D1 in the schedule time interval will be supplied by the Genco G2. Sum of the elements of any column represents the percentage of total demand of that Disco which will be supplied by the bilateral contracts. Rest of the demand will be supplied by the Poolco transactions.

In case of Poolco transaction tie-line power between area-i and area-j is settled at zero value. But in case of bilateral transition the tie-line power is not settled at zero value but settled according to the bilateral contract between Gencos of one area and Discos of other area. 


\section{Calculation of Area Control Error (ACE)}

In a practical multi area power system, a control area is interconnected to its neighboring areas with tie lines, all forming part of the overall power pool. If $P_{i j}$ is the tie line real power flow from an area-i to another area- $\mathrm{j}$ and $\mathrm{m}$ is the total number of areas, the net tie line power flow from area-i will be

$$
P_{\text {tie-i }}=\sum_{\substack{j=1 \\ j \neq i}}^{m} P_{i j}
$$

In a conventional AGC formulation, $P_{t i e-i}$ is generally maintained at a fixed value. However, in a deregulated electricity market, a Disco may have contracts with the Gencos in the same area as well as with the Gencos in other areas, too. Hence, the scheduled tie-line power of any area may change as the demand of the Disco changes. Thus, the net change in the scheduled steady-state power flow on the tie line from an area- $\mathrm{i}$ can be expressed as

$$
P_{\text {tie-new }}=\Delta P_{\text {tie- } i}+\sum_{\substack{j=1 \\ j \neq i}}^{m} D_{i j}-\sum_{\substack{j=1 \\ j \neq i}}^{m} D_{j i}
$$

Where $P_{\text {tie- } i}$, is the change in the scheduled tie-line power due to change in the demand, $D_{i j}$ is the demand of Discos in area-j from Gencos in area-i and $D_{j i}$ is the demand of Discos in area- i from Gencos in area-j.

Generally, $\Delta P_{\text {tie-i }}=0$ (Conventioanl AGC). During the transient period, at any given time, the tie-line power error is given as:

$$
\Delta P_{\text {tie-i-error }}=\Delta P_{\text {tie-i-actual }}-\Delta P_{\text {tie-i-new }}
$$

This error signal can be used to generate the Area Control Error (ACE) signal as:

$$
A C E_{i}=B_{i} \Delta f_{i}+\Delta P_{\text {tie-i-error }}
$$

Where, $\mathrm{B}_{\mathrm{i}}$ is the frequency bias factor and $\Delta \mathrm{f}_{\mathrm{i}}$ is the frequency deviation in area-i.

\section{Control System of SMES system}

When there is sudden rise in power demand in a control area, the stored energy is almost immediately released by the SMES through its power conversion system (PCS). As the governor control mechanism starts working to set the power system to the new equilibrium condition, the SMES coil stores energy back to its nominal level. Similar action happens when there is a sudden decrease in load demand. Basically, the operation speed of governor-turbine system is slow compared with that of the excitation system. As a result, fluctuations in terminal voltage can be corrected by the excitation system very quickly, but fluctuations in generated power or frequency are corrected slowly.

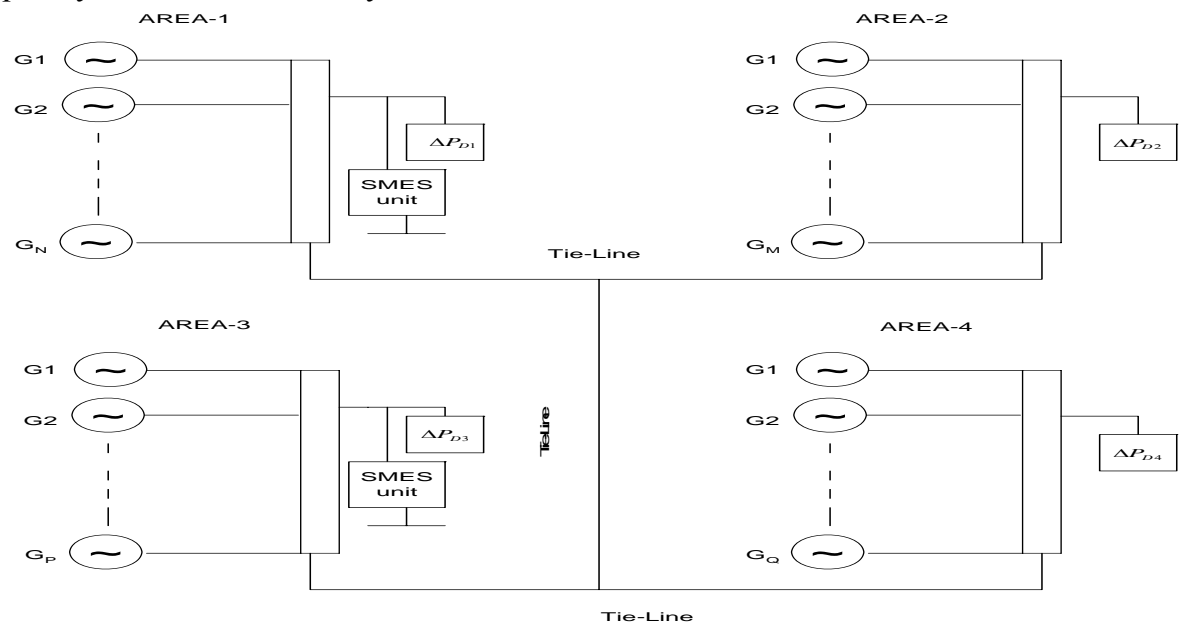

Fig. 1. Configuration of four-area interconnected power system with SMES

Fig. 1 shows the proposed configuration of SMES units in a four-area power system. Areas 1 and 3 have installed SMES1 and SMES3 in order to stabilize frequency oscillations. By controlling the active power injected/absorbed of SMES1 and SMES3, the frequency oscillations in areas 1 and 3 can be effectively damped. 
The proposed simple control scheme for SMES, which is incorporated in the two control area to reduce the instantaneous mismatch between the demand power and generation power, as shown in Fig. 2 (Joseph et al,2007), Where, $\Delta I_{d}$ is the incremental change in SMES current (kA); $T_{d c}$ is the converter time delay (Sec.); $K_{S M E S}$ is the gain of the SMES control loop for ACE signal (kV/unit ACE); $K_{i d}$ is the gain of the inductor current deviation feedgack loop $(\mathrm{kV} / \mathrm{kA}) \mathrm{L}$ is the inductance of SMES coil, $\mathrm{P}_{\mathrm{SM}}$ is Power into the inductor at any time respectively.



Fig. 2. SMES control scheme

\section{Generation Rate Constraint (GRC)}

When there is sudden rise in power demand in a control area, the stored energy is almost immediately released by the SMES through its power conversion system (PCS). As the governor control mechanism starts working to set the power system to the new equilibrium condition, the SMES coil stores energy back to its nominal level. Similar action happens when there is a sudden decrease in load demand. Basically, the operation speed of governor-turbine system is slow compared with that of the excitation system. As a result, fluctuations in terminal voltage can be corrected by the excitation system very quickly, but fluctuations in generated power or frequency are corrected slowly.

In any practical electrical power generating system, due to thermodynamic and mechanical constraints, there is a limit to the rate at which its output power can be changed. This limit is referred to as generator rate constraint (GRC). A Saturation nonlinearity is shown in Fig.3 is considered for GRC. $\mathrm{S}_{\max }$ is the maximum capacity of the generating plant. $\mathrm{S}$ is the slope representing the rate of change of generator output.

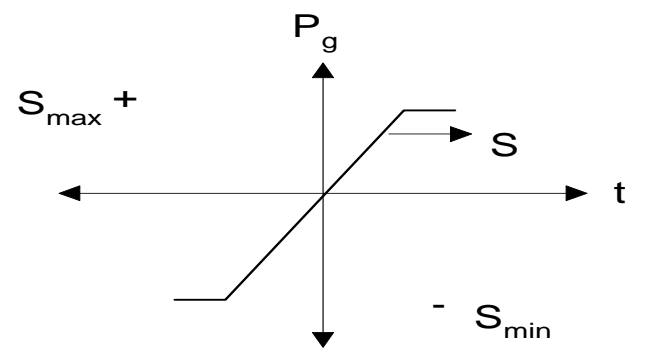

Fig. 3. Saturation Nonlinearity

The overall block diagram of AGC scheme including SMES unit and Generation Rate Constraint for an $\mathrm{i}^{\text {th }}$ area of m-area power system is shown in Fig.4.The power system block represents the power system dynamics given by $\frac{K_{p i}}{1+T_{p i}}$, where $K_{p i}$ is the system gain and is equivalent to $\frac{1}{D_{i}}$ where $D_{i}$ is the rate of change of load demand $\Delta P_{D}$ to the change in frequency $\Delta f$ and is expressed in $\mathrm{Hz} / \mathrm{pu} \mathrm{MW}$ and $T_{p i}$ is the time constant and is equivalent to $2 H_{i} /\left(f^{*} D_{i}\right)$ where, the parameter $H_{i}$ is the per -unit inertia constant.

In Fig.4 $\Delta P_{D}$ is the total demand of area-i. The part of area demand is fulfilled by bilateral transactions, and the rest of the demand will be arranged by the system operator through Poolco-based contracts. 


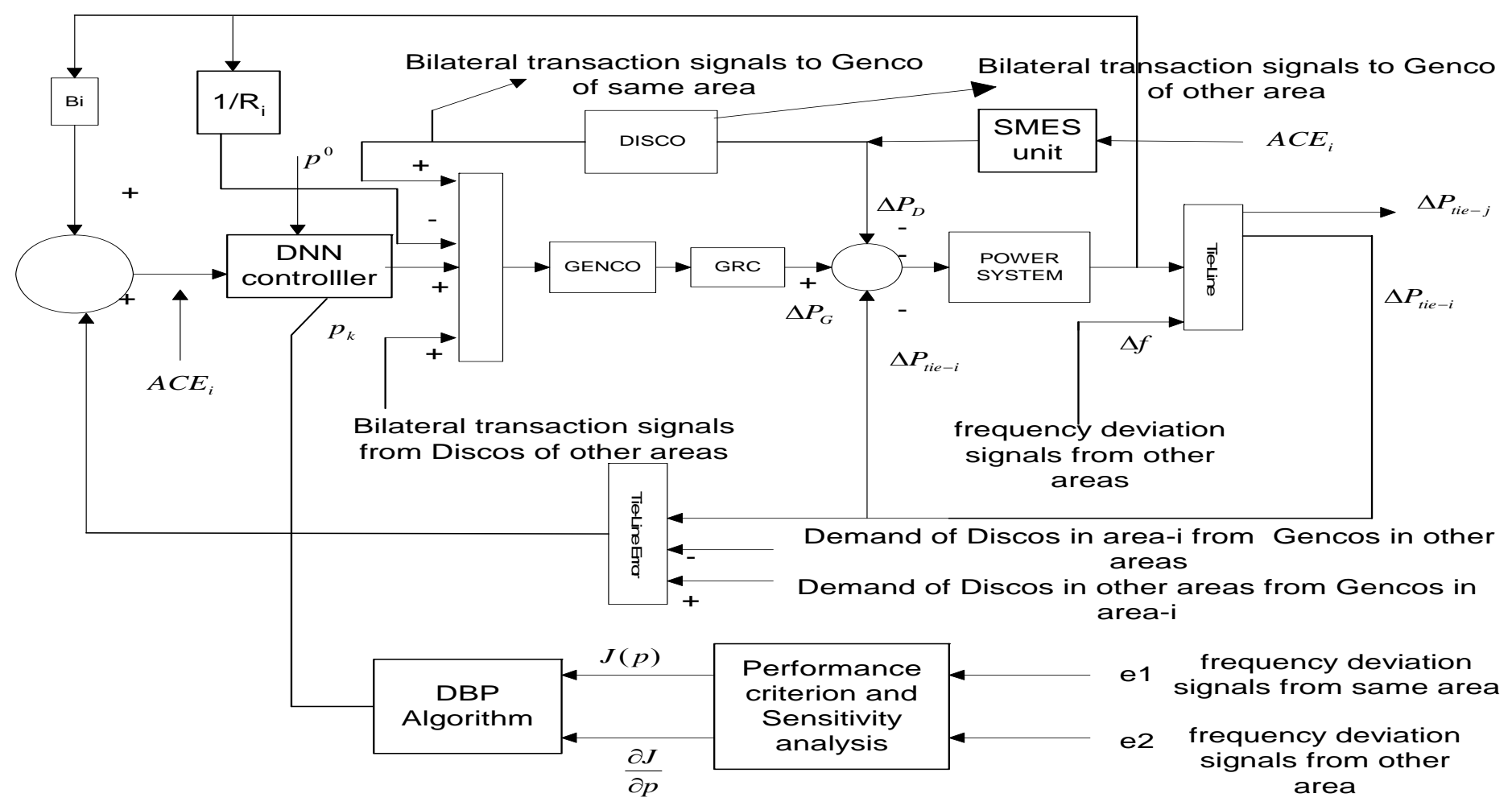

Fig. 4 AGC Block Diagram For Area-i .

\section{Dynamic Neural Network based controller design}

The input to the DNN based controller is only the ACE signal and output of controller is the signal is settled at a point according to the net Discos demand to the area. The structure of DNN based controller is given in Fig.5 below. The weight vectors of DNN based controller is found by optimizing the system by hit and trial method. The GA technique can be utilized to find out more accurate weights.

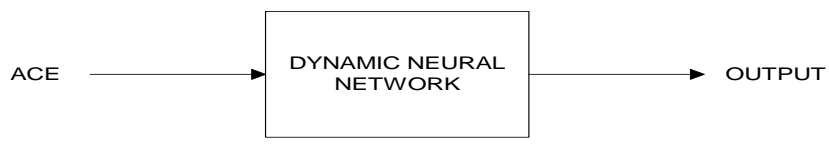

Fig. 5 DNN controller

The DNN based controller has constructed by taking only one dynamic neuron and only one unit time delay feedback output layer and input layer. There is no hidden layer considered in this design process. The advantage of DNN based controller is that it only takes $\mathrm{ACE}$ as input signal to the controller.

The DNN based controller has constructed by taking only one dynamic neuron and only one unit time delay feedback output layer and input layer. There is no hidden layer considered in this design process. The advantage of DNN based controller is that it only takes $\mathrm{ACE}$ as input signal to the controller.

In the conventional artificial neural network, many of the characteristics of its biological counterparts are ignored, like it does not take into account time delays that affect the dynamics of the system (Gupta et al, 1993). The dynamic neural network for the first time proposed by Gupta with application to control nonlinear systems in 1993 (Gupta, 2003). Dynamic Neural Units (DNU) as the basic elements of dynamic neural network receives not only external inputs but also state feedback signals from themselves and other neurons. The synaptic connections in a DNU contain a self-recurrent connection that represents a weighted feedback signal. In terms of information processing, the feedback signals involved in DNU deal with some processing of the past knowledge and store current information for future usage.

In biological systems, the electrochemical potential of each neuron is determined by the integrated effects of the entire excitatory (positive) and inhibitory (negative) postsynaptic potentials transmitted to the nerve cell for $\mathrm{n}$ integrating period of several milliseconds. A different type of neural structure has been developed by Gupta (Gupta et al, 1993), consists of positive neuron (corresponding to the excitatory portion of the neuron) and negative neuron (corresponding to the inhibitory portion of the 
neuron). This model is referred to as a positive-negative (PN) neuron and the computational model of DNN models can be written (Gupta et al, 1993) with the following equations:

$$
\begin{aligned}
& Z_{i}=\sum_{j=1}^{n} q_{i j} y_{j}, i=1,2, \ldots \ldots \ldots . . . M \\
& y_{i}=\Phi_{i}\left(x_{i}\right), i=1,2, \ldots \ldots \ldots \ldots . . . n \\
& \dot{x}=f_{i}\left(x_{i}, p\right)=\frac{1}{T_{i}}\left[-x_{x}+\sum_{j=1}^{n} w_{i j} y_{j}+\sum_{j=1}^{L} p_{i j} u_{j}+b_{i}\right]
\end{aligned}
$$

There are L time varying input signals; $\mathrm{n}$ dynamic neuron units, $\mathrm{n}$ bias terms and $\mathrm{M}$ output signals. The units have dynamics associated with them, and they receive input from themselves, bias terms and from all other units. The output of a unit $y_{i}$ is an activation function $\Phi_{i}\left(x_{i}\right)$, of a state variable $\mathrm{x}_{\mathrm{i}}$ associated with the unit. The output of the overall network is a linear weighted sum of the unit outputs. $p_{i j}$ are the input connection weights from the $j^{\text {th }}$ input to the $i^{\text {th }}$ neuron, $w_{i j}$ are the interconnection weights from the $j^{\text {th }}$ neuron to the $i^{\text {th }}$ neuron and $\mathrm{q}_{\mathrm{ij}}$ are the output connection weights from the $\mathrm{j}^{\text {th }}$ neuron to the $\mathrm{i}^{\text {th }}$ output. $\mathrm{T}_{\mathrm{i}}$ is the dynamic constant of the $i^{\text {th }}$ neuron, and $b_{i}$ is the bias term added to the unit input (or polarization) of the $i^{\text {th }}$ neuron. In the above eq. (5), the control signal $u_{j}$ taken as Area Control Error for $\mathrm{j}^{\text {th }}$ area $A C E_{i}$ and also for other area. The computational model for DNN is shown in Fig.6.

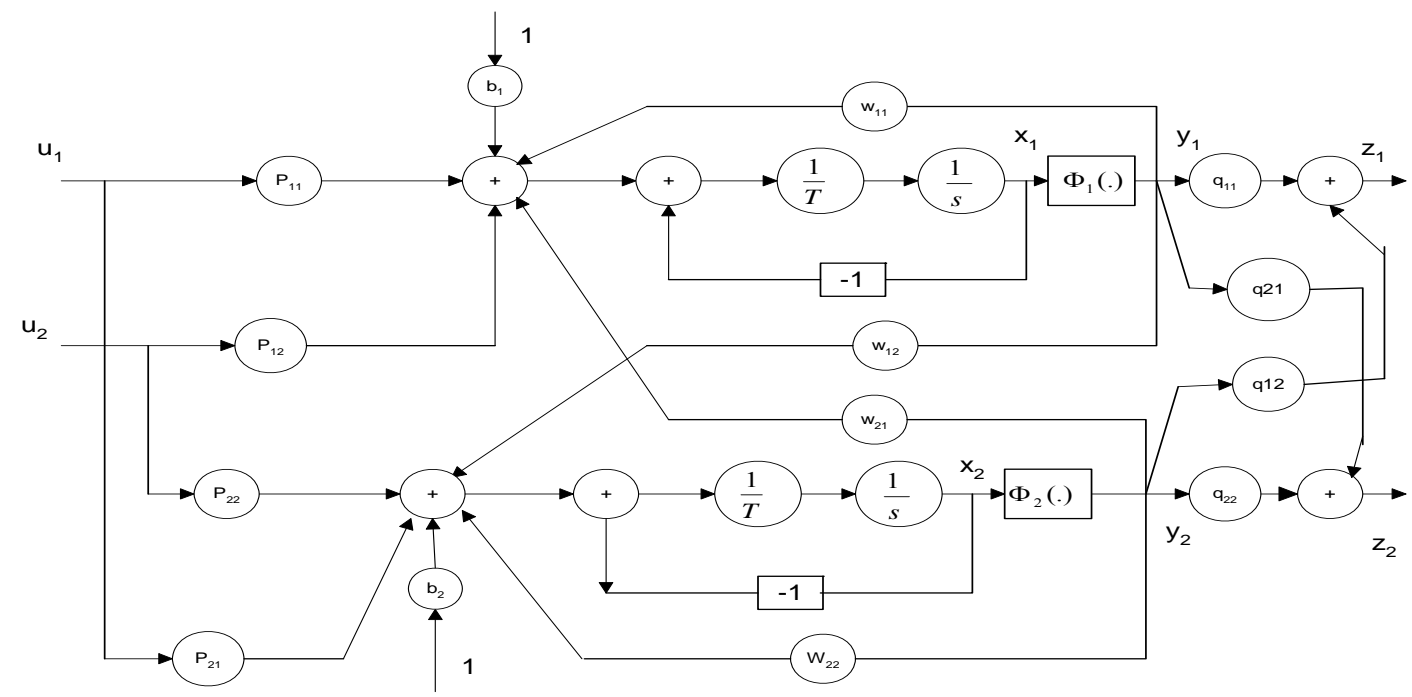

Fig. 6.The state diagram of DNN with two neurons two-input/outputs (Gupta et al, 1993).

\section{Cost Minimization Based on Dynamic Backpropogation (DBA) Algorithm}

A Dynamic Neural Network (DNN) that involves some adaptive weight learning algorithms deals with the mapping operation not only on the current input signals but also on the past history of the inputs. In the present work, the input to the DNN based controller is only the ACE signal and output of controller is the signal is settled at a point according to the net Discos demand to the area. The DNN based controller has constructed by taking only one dynamic neuron and only one unit time delay feedback output layer and input layer. There is no hidden layer considered in this design process. The DNN controller designed for each area separately.

Adaptation is based on the DNN model's training i.e. adjusting the parameters of the DNN models for load frequency control in power systems. This is done by minimizing the cost functional of the load frequency errors; ACE in the present work has been taken as shown in Fig. (4). In this paper, dynamic backpropogation learning algorithm has been used (Sabahi et al., 2007). In this study, a quadratic cost function is selected for controller design which can be expressed as (Oysal et al., 2005):

$$
E=\frac{1}{2} \int_{0}^{t}\left[z(t)-z^{d}(t)\right]^{T}\left[z(t)-z^{d}(t)\right] d t
$$

Where $e(t)=z(t)-z^{2}(t)$ is the error function for the load frequency deviations of the power system, $z(t)$ is each DNN 
model response (output) and $z^{d}(t)$ is the desired load frequency response. The required cost gradients with respect to the network parameters are calculated by adjoin sensitivity analysis based on a new dynamical system defined with adjoin state variables $\lambda_{i}$ (Oysal et al., 2005):

$$
\begin{aligned}
& -\dot{\lambda}_{i}=\frac{1}{T} \lambda_{i}+\frac{1}{T_{i}} \sum_{j} w_{i j} y_{j}^{\prime} \lambda_{j}+e_{i}(t) \sum q_{i j} y_{j}^{\prime} \lambda_{j}\left(t_{f}\right)=0 ; \\
& y_{j}^{\prime}=\frac{\partial \Phi_{j}\left(x_{j}\right)}{\partial x_{j}}
\end{aligned}
$$

Let $\mathrm{p}$ be a vector containing all network parameters. Then the cost gradient with respect to parameters ( $\mathrm{p}$ ) are given by

$$
\frac{\partial E}{\partial p}=\int_{0}^{t_{f}}\left(\frac{\partial f}{\partial p}\right)^{T} \lambda d t
$$

The weight vectors like $w$ in the equations (7) involved of DNN based controller is found by optimizing the system by hit and trial method. The GA technique can be utilized to find out more accurate weights.

\section{Test System}

The proposed scheme of DNN based PID controller for a multiarea power system, described in the previous section, and has been tested on a 75-bus Indian system (Singh et al., 1995). The 75-bus system can be divided into four control areas. The number of Gencos in the 75-bus system is given in Tables I. A general purpose Governor- Turbine model has been used, which is taken from (Anderson and Fouad, 1984).

TABLE I

\begin{tabular}{|c|c|c|}
\multicolumn{3}{|c|}{ CONTROL AREAS IN 75-BUS POWER SYSTEM } \\
\hline Control Area & $\begin{array}{l}\text { Area } \\
\text { Rating(MW) }\end{array}$ & \multicolumn{1}{c|}{ Market Participants } \\
\hline AREA-1 & 460 & Genco $1,2,3$, \\
\hline AREA-2 & 994 & Genco $4,5,6,7,8$ \\
\hline AREA-3 & 400 & Genco 9,10 \\
\hline AREA-4 & 4470 & Genco 11,12,13,14,15 \\
\hline
\end{tabular}

\section{Simulation Results}

\section{5-Bus System}

To simulate the 75-bus system, it is assumed that only the generators are participating in the frequency regulation market, Discos are not participated and both Poolco and bilateral transactions are taking place simultaneously.

To simulate the results, the change in the load demand of area-1, area-2, area-3 and area- 4 were assumed to be 0.1087 p.u (50 MW), 0.0503 p.u. (50 MW), 0.125 p.u (50MW) and 0.0224 p.u. (100 MW), respectively. Gencos' and Discos' bids of area-2 and area-4 were assumed as given in Tables II and III, respectively.

TABLE II

GENCOS BIDS IN AREA-2 OF 75-BUS SYSTEM

\begin{tabular}{|c|c|c|}
\hline GENCOS/DisCOS & PRICE(RS./KWH) & CAPACITY(MW) \\
\hline GENCO-4 & 5.0 & 25.0 \\
\hline GENCO-5 & 5.3 & 25.0 \\
\hline GENCO-6 & 4.9 & 25.0 \\
\hline GENCO-7 & 5.6 & 25.0 \\
\hline GENCO-8 & 4.7 & 25.0 \\
\hline
\end{tabular}

TABLE III

GENCOS BIDS IN AREA-4 OF 75-BUS SYSTEM

\begin{tabular}{|c|c|c|}
\hline GENCOS/DisCOS & PRICE(RS./KWH) & CAPACITY(MW) \\
\hline GENCO-11 & 4.6 & 15.0 \\
\hline GENCO-12 & 5.3 & 25.0 \\
\hline GENCO-13 & 4.9 & 25.0 \\
\hline GENCO-14 & 5.6 & 25.0 \\
\hline GENCO-15 & 4.7 & 35.0 \\
\hline
\end{tabular}


The Bilateral transactions considered between various Gencos and Discos are given below.

- The $10 \%$ power demand of area-1 is contracted with Genco-5 of area-2.

- The $10 \%$ power demand of area-2 is contracted with Genco-4 itself and 20\% power is contracted with Genco-11 of area-4.

- No bilateral transaction is considered in area-3.

- The $10 \%$ power demand of area-4 is contracted with Genco-5 of the area-2 and, 20\%by Genco- 12 of area- 4 .

The results for area-2 and area-4 frequency deviations with and without SMES unit are shown in Fig. 7(a).
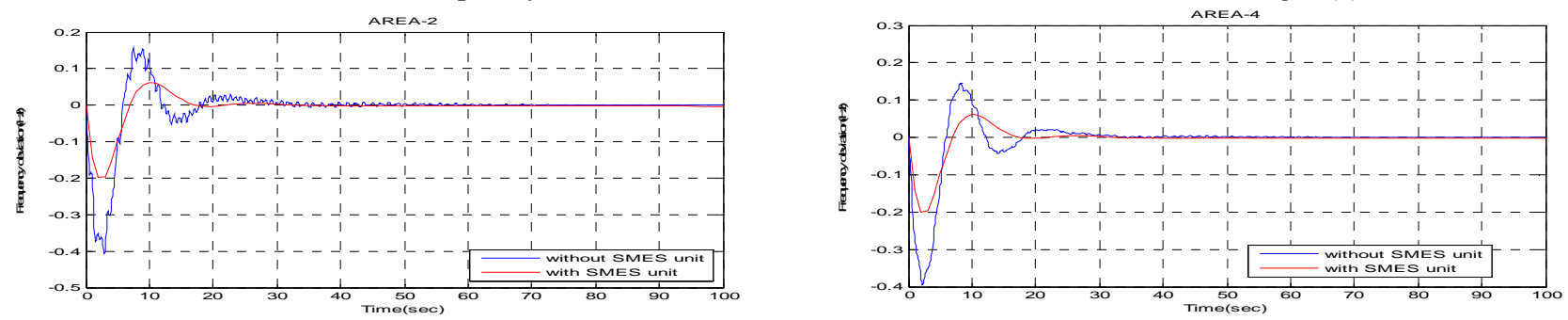

Fig. 7 (a) Frequency deviations in area-2 \& 4 for 75 -bus system

The response of the Gencos 4, 5, 6, 7 and 8 in area-2 participating in the market are also shown in Fig. 7(b). These figures also compare the performance of Gencos with and without SMES unit.


Fig. 7 (b) Response of Gencos of area-2 for 75-bus system.

Similarly, the response of the Gencos 11,12,13, 14 and 15 in area-4 participating in the market are also shown in Fig.7 (c). These figures also compare the performance of Gencos with and without SMES unit.
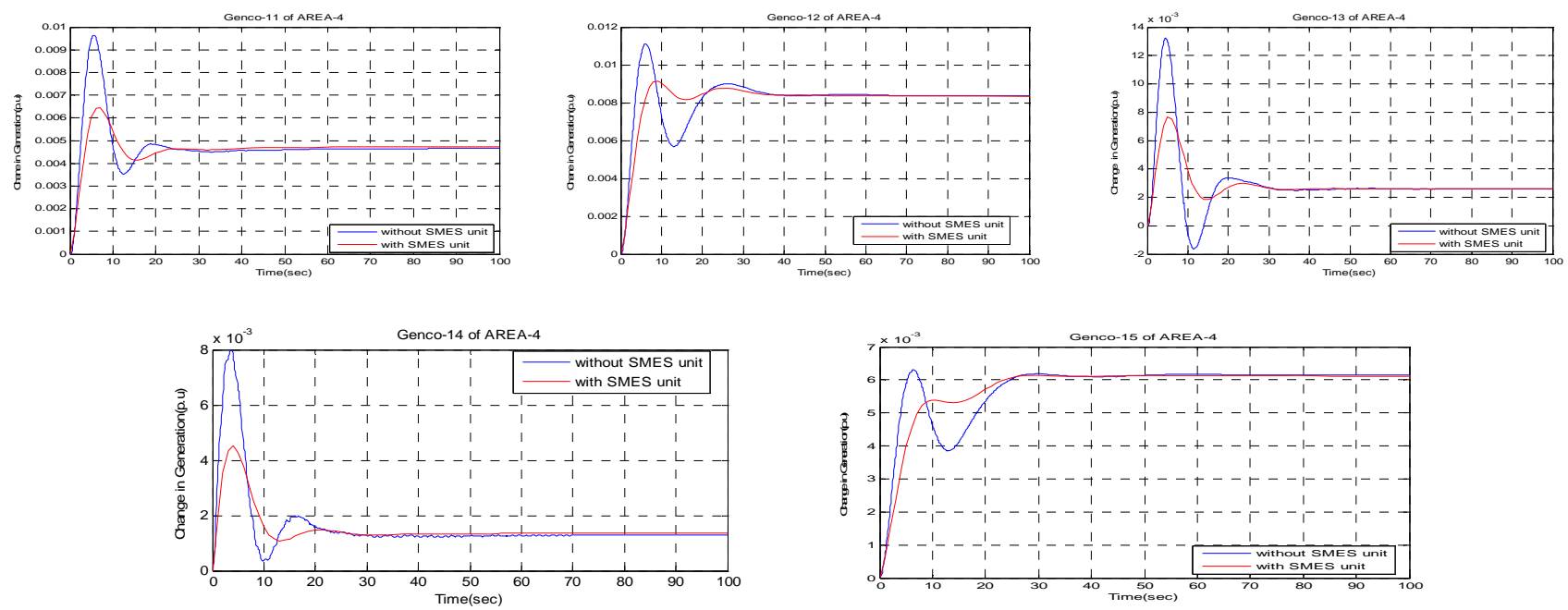

Fig. 7 (c) Response of Gencos of area-4 for 75-bus system. 


\section{Conclusions}

A Dynamic Neural Network based controller for multiarea AGC, suitable for the restructured competitive electricity market, has been proposed in this paper to meet the mixed (both poolco and bilateral) transactions. A DNN controller for each area has been developed using dynamic backpropogation learning algorithm to minimize the cost function which is taken as ACE. The proposed controller has been successfully tested on a 75-bus Indian power system for all types of load following contracts. In all the cases simulated, the area frequency error got eliminated in the steady state, and Gencos shared the increase in demand of the area, as per their participation in the frequency regulation market. It has been shown that the system frequency and tie-line power oscillations can be effectively damped out with the use of a small capacity SMES unit in either of the areas following a step load disturbance. It has also been observed that the use of ACE for the control of SMES unit substantially reduces the peak deviations of frequencies and tie-power responses. Results of the DNN based controller have been obtained with and without SMES unit. The result shows that the performance of the DNN controller with SMES unit is better than the performance without SMES unit.

\section{Nomenclature}

AGC Automatic Generation Control.

SMES Super conducting Magnetic Energy Storage unit.

DNN Dynamic Neural Network.

GRC Generator Rate Constraint.

ACE Area Control Error.

Gencos Generating companies

Disco Distribution companies

SO System operator

\section{Acknowledgement}

The authors are thankful to the Department of Electrical Engineering, Indian Institute of Technology, Roorkee, for providing the computing facilities to carry out this work. Sandeep Bhongade is grateful to G.S. Institute of Technology \& Science, Indore (M.P) and to AICTE, Government of India for sponsoring him for doctoral research work.

\section{References}

Anderson M. and Fouad A.A., 1984. Power System Control and Stability, IOWA State University Press.

Bhattacharya K., 2000. Frequency based pricing as an alternative to frequency regulation ancillary service, in Proc. Eleventh National Power Systems Conf.. Bangalore, India, pp. 210-215.

Bhattacharya K., Bhattacharya, M. , Bollen H J, 2001. Operation of Restructured Power Systems, Springer Netherlands.

Christie R.D. and Bose A., 1996. Load frequency control issues in power system operation after deregulation, IEEE Transactions on Power Systems, Vol. 11, No.3, August, pp. 1191-1200.

Donde V., Pai M.A.,and Hiskens I.A. 2001. Simulation and optimization in an AGC system after deregulation, IEEE Transactions on Power System, Vol.16, Issue 3, August, pp. 481-489.

Elgerd O. I., Electric Energy Systems Theory: An Introduction, McGraw Hill, 1982.

Elgerd O. I. and Fosha C., 1970. The Megawatt-Frequency Control Problem: A New Approach via Optimal Control Theory, IEEE Trans. Power Apparatus System, Vol. PAS-89, Apr., pp. 563-577.

Gupta M.M. and Rae D.H., 1993. Dynamic neural units with applications to the control of unknown nonlinear systems, $7^{\text {th }}$ Journal of intelligent and Fuzzy Systems, Vol. 1, No.1, pp-73-92, Jan..

Gupta M.M., Jin L., and Homma N., 2003. Static and Dynamic Neural Networks: From Fundamentals to Advanced Theory, April, Wiley-IEEE Press.

Jaleeli N., Ewart D. N., and Fink L. H., 1992. Understanding automatic generation control, IEEE Transactions on Power Systems, Vol. 7, No. 3, August, pp. 1106-1122.

Joseph R., Das D., and Patra A. 2007. Automatic generation control of an interconnected hydrothermal power system considering superconducting magnetic energy storage, Electrical Power Systems Research, Jan., pp. 572-579.

Kumar J., Nag K. and Sheble G., 1997. AGC Simulator for price-based operation part-2, IEEE Transactions on Power Systems, Vol.12, No. 2, May, pp. 527-532.

Oysal Y., 2005. A comparative study of adaptive load frequency controller designs in a power system with dynamic neural network models, Energy Conversion and Management, Vol. 46, pp. 2656-2668. 
Peterson H.A., Mohan N. and Boom R.W., 1975. Superconducting energy storage inductor-converter units for power systems, IEEE Trans. on Power Apparatus and system, Vol.PAS-94, No.4, pp-1337-1348, July/August.

Sabahi K., Nekoui M.A,, Teshnehlab M, Aliyari M. and Mansouri, M. 2007. Load frequency control in inter connected power system using modified dynamic neural networks, Control \& Automation, MED '07. Mediterranean Conference, 27-29 June, pp. $1-5$.

Shayeghi H., Jalili A., and Shayanfar H.A., 2008. A robust mixed H2/Hळ based LFC of a deregulated power system including SMES", Energy Conversion and Management, Vol. 49, pp. 2656-2668.

Singh S.N., and Srivastava S.C., 1995. Corrective action planning to achieve feasible optimal power flow solution, IEE Proc., Vol.142, Part C, November, pp-572-582.

Tripathy S.C., Balasubramaniam R., and Chanramohanan Nair P.S., 1992. Effect of superconducting material energy storage on automatic generation control considering governor deadband and boiler dynamics, IEEE Trans. on Power Systems, August, Vol. 7, No. 3, pp. 1266-1273.

\section{Biographical notes}

Sandeep Bhongade (b’1974) received M.E. degree in Electrical Engineering from V.J.T.I. Mumbai, Mumbai University (India) in 2003. Presently, he is a Research Scholar with the Electrical Engineering Department, Indian Institute of Technology, Roorkee (India). His research interests include control system, power system simulation and optimization, and electricity market deregulation.

Barjeev Tyagi (b'1964) received B. Tech. degree in Electrical Engineering from University of Roorkee (India) in 1987 and Ph. D. degree from IIT Kanpur in 2006. Presently, he is a faculty member in Electrical Engineering Department at Indian Institute of Technology, Roorkee (India). His research interests include control system, power system deregulation, power system optimization and control.

H O. Gupta (b'1950) was born in Agra, India. He received his M.E. in systems engineering and operation research and Ph.D. from the University of Roorkee in1975 and 1980 respectively. At present he is working as a professor in the Electrical Engineering Department at Indian Institute of Technology, Roorkee (India). He visited McMaster University, Hamilton, Canada, from 1981 to 1983 as a post-doctorate fellow. His research interests are in the area of computer aided design, reliability engineering, power network optimization and power transformers.

Received January 2011

Accepted March 2011

Final acceptance in revised form May 2011 